\title{
Recurrent Anaplastic Oligoastrocytoma
}

National Cancer Institute

\section{Source}

National Cancer Institute. Recurrent Anaplastic Oligoastrocytoma. NCI Thesaurus. Code C142861.

The reemergence of anaplastic olig oastrocytoma after a period of remission. 